\title{
Traffic Supervision System Using Hyg12 Load Sensors
}

\author{
N. Thirupathi Rao ${ }^{1}$, Debnath Bhattacharyya ${ }^{2}$ \\ ${ }^{1,2}$ Department of Computer Science \& Engineering, Vignan's Institute of Information \\ Technology, Visakhapatnam, AP, India \\ 1nakkathiru@gmail.com,2debnathb@gmail.com
}

\begin{abstract}
In the current paper, we present a comprehensive presentation of the transport monitoring system. The present monitoring system works with the help of count sensors, microcontroller and other units necessary to advise the drivers whoever using the busy roads. The current system works by processing the data that collects from the sensors in the ground of the roads. The primary goal of this traffic monitoring system was to collect the movement of vehicles that were moving on a selected busy road over a period and suggest the drivers take other available routes in the city such that to reduce the congestion on the city roads. Based on the number of vehicles that were on the road by crossing the sensor units were calculated and the suggestion was given to drivers whoever using such roads. Depending on the traffic signal lights of the monitoring system, the drivers can take the decision of diverting the vehicle to other possible routes such that the congestion in heavy traffic roads can be avoided such that the time travelling time of the passengers can be reduced. The results were discussed in the results section and the future scope of the traffic monitoring system was also discussed in the conclusion section.
\end{abstract}

Keywords: Traffic Monitoring, IR Sensors, 8051 Microcontroller, Congestion avoidance in traffic.

\section{Introduction}

Management of traffic issues in the real world environment is the latest research issues in the world. Several applications were being developed in the developing countries to make the traffic simple and make the passengers easy and comfort in the city for driving of vehicles [1, 2]. Traffic monitoring systems will help a lot in the administration of the traffic at various levels and also avoid the traffic problems to drivers who were travelling in the heavy traffic roads with large traffic $[3,4,5]$. By using this type of monitoring systems, transportation in the cities can be managed in terms of smarter traffic and safer traffic for the people to travel on the roads in the cities. The identification of the traffic problems on the particular roads in the city with heavy traffic roads was done and the solution to such type of problems can be avoided, which makes the drivers and the people who were travelling on the city roads will be made easy [7]. It is always better to identify the traffic problems before to the exact point of traffic jam and suggest the other vehicles on the same road to avoid the traffic jam roads such that to reduce the load on the same roads which will solve the problems to some extent.

Article history:

Received (January 25, 2019), Review Result (March 9, 2019), Accepted (July 19, 2019) 
Several sensors were available in the market for different purposes. The major sensors were aimed to use in our day to day life and for other applications in the various fields of research and other areas of real world applications [1, 2]. The type of the sensor that we have to use in our application will mainly depend on the aim of our application or the motto for which we are developing or executing our application. Different sensors will provide various values and they are intended for various uses based on their nature of the design that they intended to work. Several sensors or various sensors have various or different in our day to day life we use many different sensors. Different sensors have different determinations for work and also to support for the working of other devices. By using the sensors we can detect or identify or measure the following parameters or values like temperature, density, force, speed and body blood pressure. Several parameters were studied and observed for selecting the sensor from various available sensors in the market. Some of those parameters are the accuracy of the sensor, the working range of the sensor, the cost of the sensor and also to observe the physical environmental conditions.

\subsection{SENSORS}

In the currently proposed model of the traffic monitoring system, we had used the Hy-g12 load cell sensor for measuring the number of vehicles being crossed the point of placement of the sensors for various intervals of time. Based on the vehicles touches the place of the sensor placed under the ground, the sensor will count the number of vehicles being crossing the place or the junction. The sensor will work for a maximum number of verification intervals were 2000. The sensor was used for a various set of applications to measure the number of vehicles at various intervals of time at various capacities like 300, 500, 1000 etc [4],[ 6].

\subsection{MICROCONTROLLERS}

A Microcontroller is a small, low cost and self-contained computer-on-a-chip that can be used as an embedded system. The power consumption of these devices should always be at the level of very minimum. As the power consumption was very less, the other devices which were placed in the same device should also work so that the device should work in a good fashion or in a stable condition. Since the devices that were working with these microcontrollers are battery operated, and the devices will work with a very less amount of energy. The capacity of batteries will always be in a limited capacity as it is compared with the other sources of energy or the power supply to the devices [5].

These units are highly used in various devices and other units like consumer electronics like washing machines, micro Owens and other electronic devices, car engines for accessing and analysing the various parameters of the vehicles, various computer devices which include for saving and processing the data at various levels of devices. These devices highly suited several applications and devices which will work for long battery usage and other applications which work for very less energy consumption. These devices are characterised on the bases of a number of bits, instruction set, memory /devices, memory architecture. The microcontroller devices or units in the microcontroller are classified on the base of various bits size [6] [7] [8]. The bits in microcontroller are 8-bits, 16-bits and 32-bits microcontroller. In an 8-bit microcontroller, the size or the capacity of the internal bus is 8-bit. As the size of the internal bus was 8-bit, the Arithmetic and Logical Unit completes arithmetic and logic operations. Some of the examples of 8-bit microcontrollers used in various devices and applications are Intel 8031/8051, PICIx and Motorola MC68HC11 [9]. 
The 16-bit microcontroller was much higher and reasonable in working when compared to another set of 8-bit devices. 16-bit microcontroller devices accomplish better accuracy and performance as compared to 8-bit microcontrollers. It can be better explained with an example as follows, the 8-bit microcontroller can only use 8 bits, resulting in a final range of 0x00 $0 x F F(0-255)$ for every cycle. However, in 16-bit microcontrollers with its 16-bit data width has a range of 0x0000 - 0Xffff (0-65535) for every cycle.

Similarly, the working condition and the performance of the 32-bit microcontroller were better when compared with the previous both the controllers. The 32-bit instructions will be well executed and implemented in the case of performing arithmetic and logical operations. These microcontrollers are highly used in mechanically controlled devices, including implantable medical devices and embedded systems. Some of the examples of the 32-bit microcontroller devices and units are Intel/Atmel 251, PIC3x. The microcontroller devices are classified into two types based on the memory that they use whenever these units are used in several gadgets. They are,

Embedded memory microcontroller.

External memory microcontroller

\subsubsection{EMBEDDED MEMORY MICROCONTROLLER:}

Whenever a microcontroller unit was fixed on an embedded unit which was having all the practical blocks that were available on a single chip is called an embedded microcontroller. The example for an embedded memory microcontroller was 8051 microcontroller having a program and data memory, I/O ports, serial communication, counters and timers and interrupts on the chip is an embedded microcontroller.

\subsubsection{EXTERNAL MEMORY MICROCONTROLLER:}

Whenever a microcontroller unit was not built on a single chip and also on the single device or single board, and the unit was fixed with all functional units not on the same device or on the same unit such devices are called or known as the external memory microcontroller. The best example of this type of memory units or the functioning points of the unit is the 8031 microcontroller unit which was having no program memory on the chip.

\subsection{CLASSIFICATION ACCORDING TO INSTRUCTION SET}

The instruction set was the most important concept of the microcontrollers for operating the devices in which the unit was developed and embedded in it. To make the instructions, the following are the set of instructions. They are as follows,

CISC: CISC is Complex Instruction Set Computer. It permits the programmer and the user to use one instruction in place of much simpler instruction.

RISC: The RISC stands for Reduced Instruction Set Computer, this type of instruction sets lessens the strategy of the microprocessor for engineering, commerce and industrial standards. It allows each instruction to operate on any register or use any addressing mode and simultaneous access of program and data.

\subsection{MICROCONTROLLERS USED IN THE TRAFFIC MONITORING UNIT}

8051 Microcontroller is one of the mostly used microcontrollers that were available in the market today for the usage of various types of users for a various set of applications. Due to the more applications size and heavy usage and the various set of instructions and furthermore sizes 
that supported by the microcontroller was the reason. This microcontroller was available in three sizes in the market today. Those sizes are Short, Standard, Extended. It has $4 \mathrm{~KB}$ chip program memory. It has 128 bytes RAM, four register banks, 16-bit address bus, 16-bit timers, four 8-bit ports and 16-bit program counter and data pointer.

\section{LITERATURE REVIEW}

Xiao had proposed a traffic monitoring system such that to lessen the city traffic strain, decrease the delay time of vehicles on the road. This paper explains the traffic monitoring system based on RFID. Integrated climate, road conditions and power control all the factors considered, this papers choose to use passive tags to identify the vehicle. Multifunctional integration RFID reader read the tag information stored within the vehicle efficiently and sends it to the data processing centre. The system according to the real-time data processing results realises the monitoring purpose of the intersection vehicle.

Sagar sukode proposed a new context-aware method to find the existing position and density of traffic and active supervision of traffic signals along with the ecological circumstances. To make easy the proposed targets they proposed an architecture of Vehicle Traffic Congestion Control \& Monitoring System in IoT. This architecture is alienated into two main modules i.e. hardware module and software module. The proposed scheme makes use of new technologies for collection, association and broadcast of data to give a competent and precise opinion of traffic solidity by which traffic-aware applications can exploit the users.

Satya Priya Biswas et al., had presented a variety of approaches made to improve the traffic system across the world. A relative reading has been made of various possible researches in which intelligent traffic system (ITS) emerges as a significant submission region. Significant key points of every research are painted and judged on the source of applying them to mounting countries like India. Representation is also projected which uses infrared proximity sensors and a centrally placed microcontroller and uses vehicular length along a length to implement intelligent traffic monitoring system.

Mahendra Dharmar et al., had discussed the movement familiarity with the driver is one of the prime concentration as far as a person on foot and street security. Driver encounter assumes a noteworthy part and driving requires careful consideration regarding changing conditions both inside and outside the vehicle. Any slip by in driver consideration from the essential errand of driving could conceivably prompt a mishap. It is watched that, the absence of consideration on the continuous movement and uninformed about the activity data, for example, activity lights, street signs, activity tenets and directions are the real reason for the vehicle crash. Movement signals and signage are the most appropriate decision of activity control for the crossing point, guarantee that driver can see the data far from the convergence with the goal that he/she can stop securely after survey the yellow and red show. At that point, after survey the flag operations and conditions the driver can stop his/her vehicle effectively before entering the convergence.

From the above literature review and the various previous works, it is observed that the use of sensors for identifying the movement of vehicles was significant on roads such that to reduce the congestion on busy city roads. However, counting the number of vehicles being crossing a particular point of location on roads for counting the vehicles was not observed. Hence, we took this problem and considered the count sensors to be used to count the number of vehicles being crossing the particular signal point over a period. By counting the number of vehicles, we will display the drivers on the same road to take necessary steps such that to avoid the traffic congestion on busy city roads at peak hours of the time. The sensors considered here in the 
current work were the weight sensors such that in most of the previous works very few people had used the weight sensors. The chance of missing vehicles by using weight sensors was decidedly less compared to the other types of sensors for calculating or counting the number of vehicles were crossing the road.

\section{DESIGN OF THE TRAFFIC MONITORING MODEL}

The design of the traffic model was placed as follows. The basic model was the layout designed in such a way that the two sensors were placed in the two lanes on the roads. Each sensor was placed on each lane, and the sensor detects the movement of the vehicle regarding the density that is the count of the vehicle. The count of the vehicle was taken as the minimum level. The basic count of each vehicle was taken as $800 \mathrm{Kgs}$. The sensor will detect the count of the vehicle only those vehicles the count is more than $800 \mathrm{Kgs}$. Each sensor will detect the count of the vehicle for a certain period and the count of the vehicles were made and the data being sent to the sensor network that was established near to the side of a road and the network will send the data to the next point of the traffic signal such that to inform the status of traffic. Based on the traffic level, the vehicles were continued on the same road or the same direction of the road or the direction of the vehicles were directed to other roads or another set of roads for further reduction of traffic in the existing roads in the city.

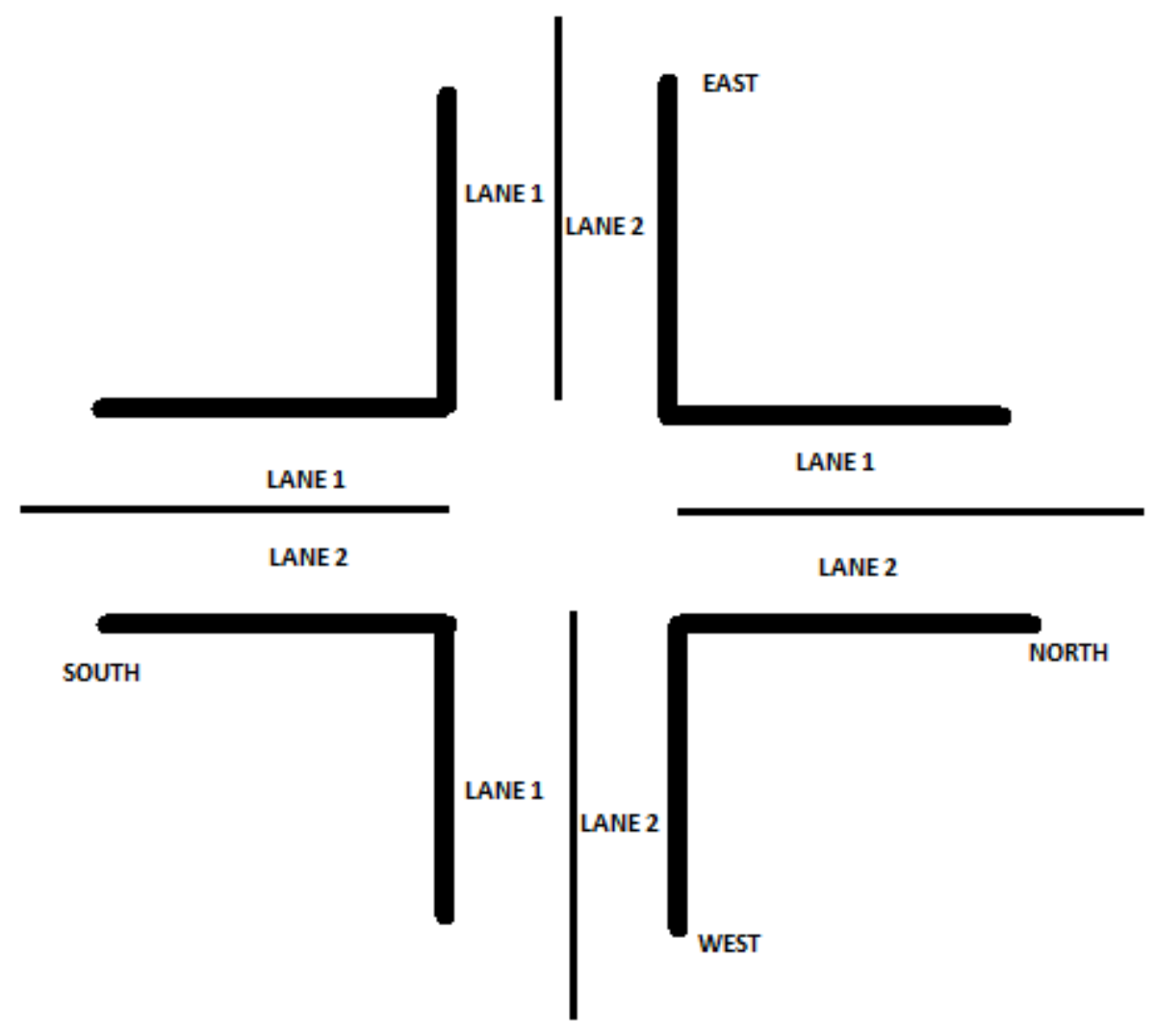

Figure 1. The Circuit Layout of the Traffic Monitoring System 
The layout model of the total architecture of the traffic monitoring system was given in below figure. The count of the vehicles will be calculated regarding the density of the vehicles. The density can be calculated as the number of vehicles was travelling through these sensors over a point of time, or it may also be considered as for a particular period. The density of the vehicle movement can be calculated by using an Infra-Red sensor. There are several sensors available in the market for the calculation of the density of the vehicles.

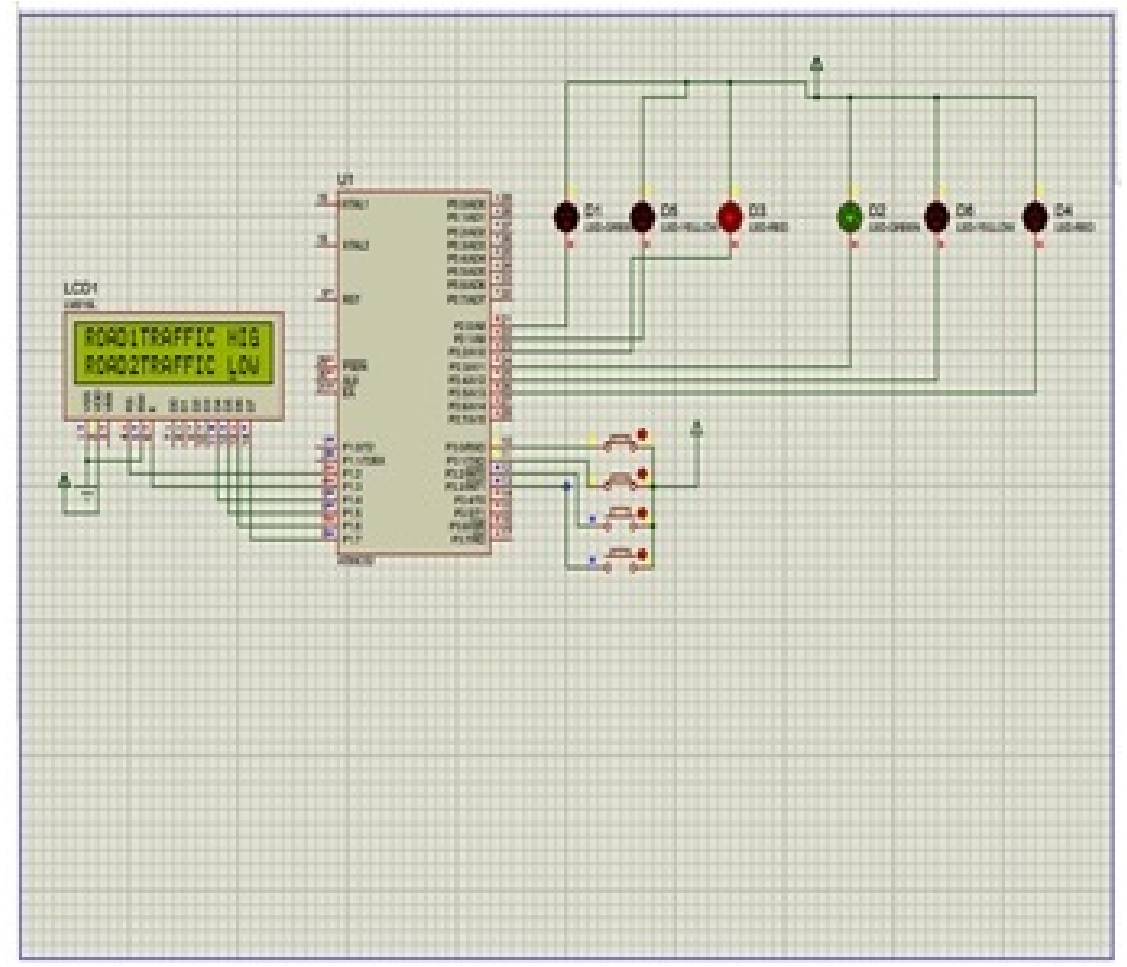

Figure 2. Layout model of the Traffic Monitoring System

For the current proposed system, four sensors were being used for which all the sensors were fixed at two roads with two lanes in each road. The microcontroller unit that was arranged in the kit of the system was the 8051 microcontroller unit. To signal the vehicles that were being gone on the roads, around six LEDs were collected such that the two lights are green in colour, two lights are in red colour, and the other two lights are in yellow colour. In order to work with the system and to operate the system without the interference of human beings, an automated program was being developed such that the machine will operate the system without interruption of the human being. Programming was developed such that the total circuitry and the total kit of the system was being developed in both the microcontroller programming for the working of the 8051 microcontroller and also the $\mathrm{C}$ programming with interface model and other interfacing units for users and the operators to intervene with the system to make corrections if any needed in the existing system or in the coming future. The user interface that was being developed in the system was very easy to use and can be understood easily by any sort of employees who were working in the real-time environment. For the microcontroller, four buses for the data transfer and also for the processing of the data and also to supply commands to the other units was done through the programming mode of the microcontroller 8051. 
The data that was being collected from the sensors which were placed on the roads will be directly sent to the system that was placed on the side of the road. The system will count the vehicles with the amount of count the vehicles are being crossed through these sensors for a point of time. The time taken to count the count of the vehicles was not static, it is dynamic. The time will be considered based on the timings of them, i.e., the number of vehicles that were being crossed through these sensors was collected and calculated. The output of the traffic monitoring system LED lights were placed at the signal points that were located before 3 to 4 kilometres from the present signal point at where the actual monitoring system was located. When the data was being collected, the sensors will collect data in terms of the count of the vehicle. We had assumed the initial count that could be estimated as the heavy traffic was nearly 200 to 450 vehicles, but during the heavy traffic timings, the number was reduced further from 100 to 180 vehicles such that to decrease the over traffic burden on the roads by early prediction of the traffic.

In general, the regular traffic timings will be considered the number of vehicles should be 400 to 850. If the density of vehicles data increases from the sensors, the monitoring system will give as an output in the form of normal traffic. The traffic levels that were taken as three types of representation to the drivers on roads who were using the road. If the count calculated from the sensors was in the form of regular case, then the green light will be represented to the drivers at the before signal point such that the drivers will have the choice to operate the vehicle in the possible other routes if required. If the count density was heavy, the monitoring system would display the drivers that the lights were in red and the drivers whoever driving in the same road may take their own decisions and may choose to some other possible routes. At some times the time taken for calculating the count of the vehicles was sometimes it was static, and at sometimes it was in dynamic nature. Based on the requirements of the user, the settings in the system can be made from time to time. The density calculation range may also be possible to change from time to time based on the requirements of the user.

A vehicle driver can use the information that was collected from various sensors do reach the destination in a faster and smarter way. We place sensors on the road. Every sensor has a transmitter and a receiver. The signals between the transmitter and receiver are used to detect the traffic density. Whenever a vehicle passes on the road the signals between transmitter and receiver will get disturbed, and density will be noted. We place these sensors on the roads which have two lanes. We consider both the lanes individually. We consider the starting point for each lane and our endpoints are 600 meters away from our starting point. This 600 meters distance is divided into three regions of 200 meters each. 0-200 meters is the first division and one sensor is placed in this region. 200-400 meters is the second division and the second sensor is placed in this region. 400-600 meters is the third division. We use LEDs to display the traffic status in a particular lane. By default, we have green LEDs glowing this represents that the lane has low traffic density. Whenever density is recorded in the third division and as well as in our second division this represents medium density. Green led will stop glowing and yellow led starts glowing. Moreover, in case whenever density is recorded in all first, second and third divisions, it represents high density. In this case, red led starts glowing and other two LEDs will be stopped. According to this, the vehicle driver can opt for any lane to reach his destination.

\section{RESULTS}

The traffic monitoring system was being implemented with different sensors at different places on the road at various time slots. Based on the count of the vehicles, the IR sensor will calculate the number of vehicles that were crossing the sensors and the data will be supplied to 
the system which was connected to it for further processing. Once the data was being collected from the sensors, the decisions will be given by the system after processing the data that was received from the sensors. The data that was retrieved from the sensors at various time slots and the decisions that were being guided by the system based on the input data was tabulated as follows,

Table 1. Results observed from the proposed system at various time intervals,

\begin{tabular}{|c|c|c|c|}
\hline Timeslots & Duration of the slot & $\begin{array}{c}\text { Count of the Vehicles } \\
\text { (Vehicles) }\end{array}$ & $\begin{array}{c}\text { The decision for } \\
\text { the driver }\end{array}$ \\
\hline 01 & 16 seconds & 90 & Yellow \\
\hline 02 & 10 seconds & 120 & Yellow \\
\hline 03 & 29 seconds & 321 & Red \\
\hline 04 & 45 seconds & 512 & Red \\
\hline 05 & 09 seconds & 458 & Green \\
\hline 06 & 55 seconds & 254 & Green \\
\hline 07 & 60 seconds & 658 & \\
\hline
\end{tabular}

Here from the above table, the results for various timeslots with various weights of the vehicles were observed and tabulated. Different timeslots for calculating the vehicle movement at different levels of traffic on roads were observed and calculated. The data was collected at various levels at the general leisure time of the roads and the heavy traffic time of the roads and at night times after $10 \mathrm{PM}$ also recorded the values and was tabulated. Based on the values observed from the sensors, appropriate decisions were taken and the results and the decisions were passed to the signal points which were placed before the point of the signal junction so that the congestion in road traffic can be avoided.

\section{CONCLUSIONS}

In the current article, the traffic monitoring system which was developed and tested in various cases and results were noted. The testing of the system was done by implementing it on the road, and the results were taken to display the LED lights that were placed on the roads at a distance of 3 to 4 kilometres away from the actual place of the monitoring system. As a result, drivers whoever is driving in the same road might identify the signals that were being placed in the form of green, red and yellow and the drivers will have a choice to choose the other available routes to reach the destination smoothly. In the present system, the count of the vehicles will be calculated, but the extension can be made to calculate a number of vehicles with their weights by increasing the capacity of the sensors and also the capacity to store the data at the system. However, the system was being implemented by writing $\mathrm{C}$ and microcontroller programming and in the future, these can be upgraded to some more fixed or some new languages or software's which were exclusively for this monitoring system. The goal of the current proposed system can be solved much more precisely in the future by using more number of sensors and more devices with the latest software technologies such that the traffic problems on the Indian roads can be reduced to some extent. 


\section{References}

[1] Klausner, A., Erb, S., Tengg, A., "DSP Based Acoustic Vehicle Classification for Multi-Sensor Real-Time Traffic”. Graz University of Technology, Graz, Austria. (2007)

[2] Forren, J. F., Jaarsma, D.: “Traffic Monitoring by Tire Noise”. Proc. IEEE Conf. on Intelligent Transportation System, Boston, MA, Nov (1997), pp. 177-182. DOI: 10.1109/ITSC.1997.660471

[3] Ding, J., Cheung, S.Y., Tan, C.-W., Varaiya, P.: “Signal processing of sensor node data for vehicle detection”. Seventh International IEEE Conference on Intelligent Transport System. (2004) DOI: 10.1109/ITSC.2004.1398874

[4] Lopez-Valcarce, R., Mosquera, C., Perez-Gonzalez, "Estimation of road vehicle speed using two omnidirectional microphones: A maximum likelihood approach”, EURASIP Journal of Applied Signal Process. (2004) DOI: 10.1155/S1687617204311133

[5] Lo, K. W., Ferguson, B. G.: "Broadband passive acoustic technique for target motion parameter estimation", IEEE Transactions on Aerospace Electrical Systems, vol. 36, pp.163, (2000). DOI: 10.1109/7.826319

[6] Cheung, S., Coleri, S., Varaiya, “Traffic Surveillance with Wireless Magnetic Sensors”. University of California, Berkley. USA. (2005)

[7] Nellore Harika , Muthyala Vamsilatha, Nakka Thirupathi Rao, Debnath Bhattacharyya and Tai-hoon Kim. "Performance Analysis and Implementation of Traffic Monitoring System using Wireless Sensor Network for reducing blockage in Traffic on Indian City Roads”. Journal of Statistical Computing and Algorithm. Vol. 1. No. 1. Dec. (2017). GVPress. pp.15-26.

[8] Jeong-Joon Kim, Yong-Soo Lee, Jin-Yong Moon, Jeong-Min Park.Bigdata based Network Traffic Feature Extraction.International Journal of Communication Technology for Social Networking Services . Vol. 6. No. 1. Mar. (2018). GVPress. pp:1-6.

[9] N. Thirupathi Rao and Debnath Bhattacharyya, "Sensor Based Traffic Monitoring System on Indian City Roads", International Journal of Transportation, SERSC Australia, ISSN: 2287-7940 (Print); 2207-6433 (Online), vol.6, no.3, December, (2018), pp. 1-10. 
Traffic Supervision System Using Hyg12 Load Sensors 